\title{
Segmental Arterial Mediolysis (SAM) Leading to Chronic Renal Insufficiency
}

\author{
John-Paul O'Shea (1D \\ Sarah Gordon ${ }^{2}$ \\ Richard Horak ${ }^{3}$ \\ J Matthew Meadows ${ }^{3}$ \\ 'Department of Medicine, Tripler Army \\ Medical Center, Honolulu, HI, 96859, \\ USA; ${ }^{2}$ Department of Nephrology, \\ Walter Reed National Military Medical \\ Center, Bethesda, MD, 20889, USA; \\ ${ }^{3}$ Department of Radiology, Tripler Army \\ Medical Center, Honolulu, HI, \\ 96859, USA
}

\begin{abstract}
Background: Segmental arterial mediolysis (SAM) is a rare self-limiting nonatherosclerotic, non-inflammatory vasculopathy. SAM typically affects the visceral arteries of the abdomen to include the celiac, mesenteric, and renal arteries. SAM has a favorable prognosis in most cases with an asymptomatic course but can have mortality rates as high as $50 \%$ due to acute aneurysmal rupture. Very few cases of adverse long-term sequelae involving SAM have been described, and this report of chronic kidney disease represents a sentinel case illustrating that chronic disease can and does occur as a result of SAM and should be investigated for at follow-up.
\end{abstract}

Case Presentation: In this case report, we describe a case of a 45 -year-old male with erectile dysfunction but without any readily identifiable risk factors for chronic kidney disease (CKD) or vasculopathy, who presented with bilateral renal infarction and parenchymal infarcts due to SAM and who subsequently developed CKD at follow-up. We conduct a mini-literature review that discusses the pathogenesis of SAM in the context of vasospastic diseases, as well as compares the outcomes of observation-only, versus medicalmanagement, versus endovascular-interventions in patients with SAM.

Conclusion: This is the first case to our knowledge of CKD occurring as an outcome of SAM without any preceding significant comorbidity, highlighting that whereas SAM is of itself rare and typically resolves, chronic disease can linger and should be evaluated for on follow-up. Further, we argue that radiological evidence of precursor vasospastic disease may exist in several locations apart from the index lesion and thus warrants wider whole-body radiographic exploration for lesions as an opportunity to prevent chronic sequelae as illustrated in this case report from occurring. Finally, a review of published case-series suggests that disease progression is less likely to occur after endovascular-intervention compared to observation-only or medical management and the risk of intervention vs conservative management should therefore be discussed with the patient.

Keywords: vasospastic, vasculopathy, erectile dysfunction

\section{Background}

Segmental Arterial Mediolysis $(\mathrm{SAM})$ is a rare $(\sim 1 / 100,000)$ self-limiting nonatherosclerotic, non-inflammatory vasculopathy that typically affects the medium and large visceral arteries of the abdomen to include the celiac, mesenteric and renal arteries, and typically has a favorable prognosis when patients overcome the acute vascular insult. ${ }^{1,4}$ The pathophysiology of SAM includes both an acute phase and a reparative phase. During the acute phase, symptoms include severe abdominal and/or flank pain or headaches due to dissection and aneurysmal rupture or infarction of the vascular beds. ${ }^{2}$ Acute intra-abdominal or retroperitoneal hemorrhage share the main cause of increased mortality $(20-50 \%)$ observed in the acute
Correspondence: John-Paul O’Shea Department of Medicine, Tripler Army Medical Center, 99-57I Pohue St, Aiea,

Honolulu, $\mathrm{HI}, 9670 \mathrm{I}$, USA

$\mathrm{Tel}+$ I 5049868573

Email john-paul.oshea.civ@mail.mil 
phase. ${ }^{1,3}$ The most common long-term outcome of SAM reported in the literature is hypertension, but otherwise there are no other reports of significant chronic sequelae of this condition.

Here we report a case of a 45-year-old male patient with no past medical history and normal baseline renal function who developed stage 3 Chronic Kidney Disease (CKD) after sustaining bilateral renal artery dissections secondary to SAM. This is the first reported case of CKD following SAM without prior comorbidities.

\section{Case Presentation}

A 45-year-old Caucasian male presented to the Emergency Department following 3 days of progressively worsening right lower quadrant abdominal pain and headaches. His past medical history was remarkable for long-standing back pain and erectile dysfunction. The patient was a social drinker and occasionally smoked. Initial clinical workup was unremarkable with normal blood pressure and renal function (BP 124/86; serum creatinine was $1.0 \mathrm{mg}$ / $\mathrm{dL}$ with an eGFR of $90 \mathrm{~mL} / \mathrm{min} / 1.73 \mathrm{~m}^{2}$ ). A CT scan of the abdomen and pelvis was performed with intravenous contrast was performed per local protocol, revealing a right lower pole renal infarct. There was also perivascular inflammation involving the celiac artery. Initially a thrombotic/hypercoagulable state was hypothesized as the etiology. An ECG, transthoracic echocardiography bubble studies, d-dimer and coagulation studies were performed with no abnormal findings. Serologic studies evaluating for systemic vasculitis including ESR, CRP, ANCA and ANA were also negative. The patient was admitted for 3 days and was discharged on apixaban $5 \mathrm{mg}$ once daily with stable creatinine and eGFR.

The patient represented to the ED 16 days later with recurrent right flank pain, blood pressure $151 / 88 \mathrm{mmHg}$, temperature $36.7^{\circ} \mathrm{C}$, serum creatinine of $1.3 \mathrm{mg} / \mathrm{dL}$, eGFR $65.7 \mathrm{~mL} / \mathrm{min} / 1.73 \mathrm{~m}^{2}$, ESR $32 \mathrm{~mm} / \mathrm{h}$, CRP $12.32 \mathrm{mg} / \mathrm{dL}$. Urinalysis was bland with no granular or epithelial casts. A CT angiogram of the aorta was performed to evaluate for aortic dissection, which revealed progression of the previously seen right renal infarct, now extending into the mid pole. There was also an acute dissection of the anterior and posterior divisions of the right renal artery, with several smaller branch occlusions in the mid and lower pole. In addition, there was new perivascular inflammation involving the main right renal artery and persistent inflammation involving the celiac artery, but no dissection or end organ damage. Interventional radiology was consulted for management of the renal artery thrombus to prevent worsening of the infarct. The patient was taken for a renal artery angiogram which confirmed the presence of a dissection and multiple segmental and smaller branch occlusion in the mid to lower right kidney. A decision was made to initiate catheter-directed thrombolysis, and the patient was monitored in the ICU overnight per protocol. Within 48 hours after the initiation of catheter directed thrombolysis, suffered fever and urinalysis was leukocyte esterase and nitrite positive and had $2+$ blood and $2+$ protein and was subsequently managed with Cefepime for urinary tract infection although urine culture ultimately showed no growth. The following day, the patient returned to IR for a repeat angiogram which revealed no significant reduction in arterial thrombus burden and a persistent dissection. Due to the patient having normal blood pressure, and significant pain improvement, thrombolytic therapy was discontinued and the patient was initiated on dual antiplatelet therapy with aspirin $(81 \mathrm{mg}$ daily) and clopidogrel (75 $\mathrm{mg}$ daily). At the point of discharge, the $\mathrm{sCr}$ was $1.0 \mathrm{mg} / \mathrm{dL}$, eGFR $89.0 \mathrm{~mL} /$ $\min / 1.73 \mathrm{~m}^{2}$.

Twelve days later, the patient was readmitted, at that time complaining of gross hematuria and passing tissue on urination with elevated creatinine $(1.63 \mathrm{mg} / \mathrm{dL})$ and eGFR $\left(50.1 \mathrm{~mL} / \mathrm{min} / 1.73 \mathrm{~m}^{2}\right)$. A CT angiogram of the renal arteries was performed, showing a new left lower pole renal infarct and a left renal artery segmental branch dissection (Figure 1). There was a similar appearance to the perivascular inflammation involving the celiac and right renal artery branches with no increase in right renal infarct size. Genetic testing panels including COL3A1 for collagen disorders yielded nothing.

At that time other contributors to the acute kidney injury and gross hematuria, including variously: hypovolemia; post-renal obstructive causes; glomerular and tubulointerstitial nephropathies (eg, contrast-induced nephropathy, acute interstitial nephropathy, acute tubular necrosis; were considered but appeared unlikely given lack of obstruction found on imaging (renal ultrasound and CT) as well as profuse red blood cells and protein on urinalysis but otherwise bland urine microscopy). There was no albuminuria. Throughout his presentation, the patient maintained adequate urine output $\sim 120-200 \mathrm{~mL} /$ hour (body mass $95.7 \mathrm{~kg}$ ). At this stage and in light of the radiographic abnormalities including inflammatory changes involving the celiac and renal arteries with associated bilateral renal artery dissections and the absence of vasculitic-, collagen 


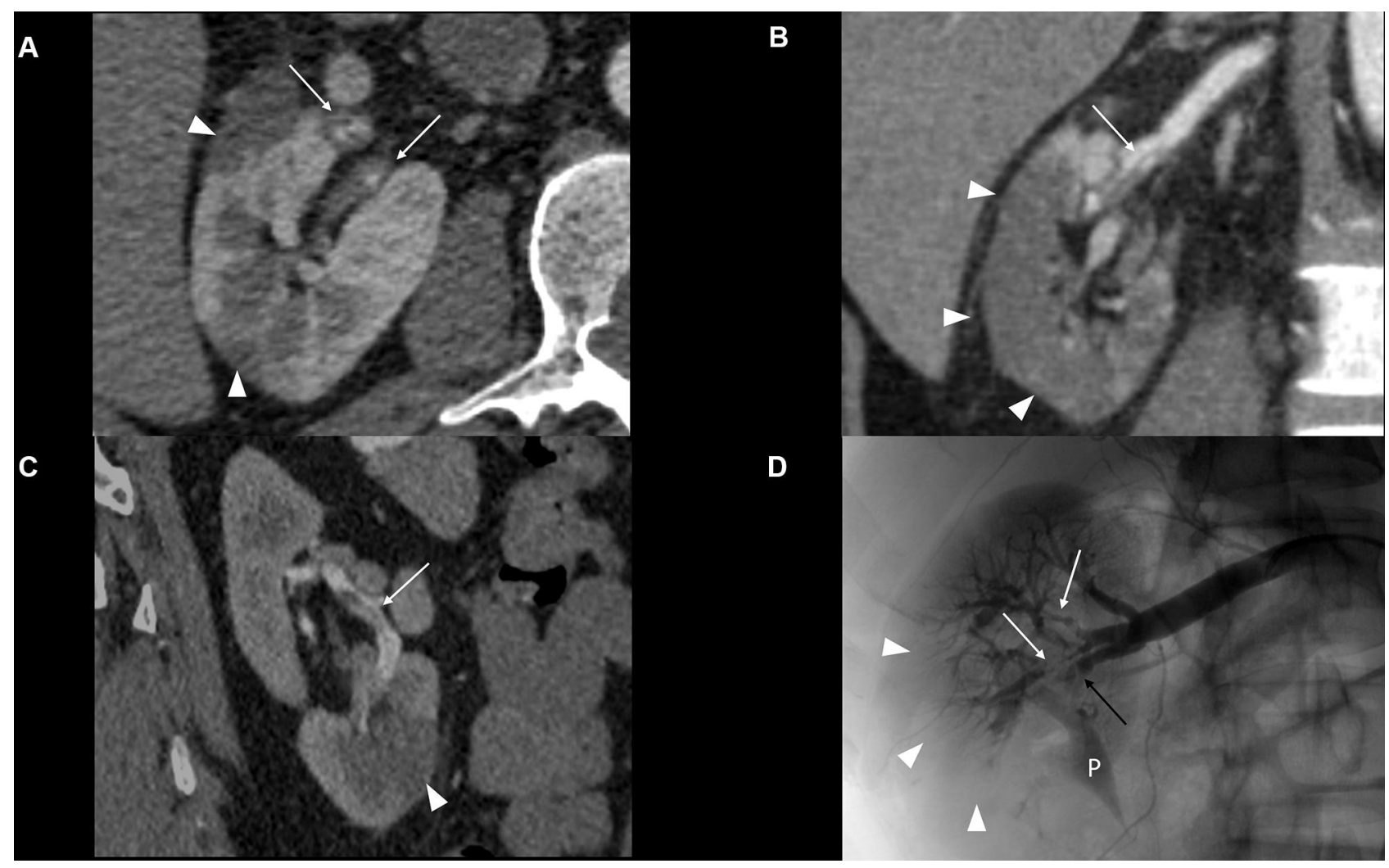

Figure I CT angiogram images, demonstrating bilateral renal artery lobar branch dissections (white arrows) on the right (A and B) and left (C), as well as associated parenchymal infarcts (white arrowheads). Digital subtraction angiogram of the right renal artery (D) demonstrating lobar and segmental branch dissections (white arrows), segmental branch occlusion (black arrow) and infarcted parenchyma (white arrowheads). Excreted contrast is also noted in the renal pelvis (P).

defect-, or thrombophilia-markers, the provisional diagnosis was a non-atherosclerotic non-inflammatory vasculopathy.

As there was no indication for surgical or additional endovascular management, the multidisciplinary team decided to manage the patient medically with atorvastatin (80 $\mathrm{mg}$ daily), Aspirin (81 $\mathrm{mg}$ daily), clopidogrel (75 mg daily), metoprolol (25 mg daily), amlodipine ( $5 \mathrm{mg}$ daily) with blood pressure goals of $<130 / 80$ and counseling to adhere to a low sodium diet, home blood pressure monitoring and avoidance non-steroidal antiinflammatories.

Within 2 weeks of this latter presentation, hematuria had been resolved, there was no proteinuria or albuminuria on urinalysis; however, serum creatinine remained elevated and eGFR depressed. At 15-month follow-up, his kidney function had stabilized with elevated creatinine $(1.3-1.5 \mathrm{mg} / \mathrm{dL})$ and eGR $\left(58-63 \mathrm{~mL} / \mathrm{min} / 1.73 \mathrm{~m}^{2}\right)$ levels meeting the KDIGO criterion for CKD stage G2/3. A Renal CTA at this time demonstrated chronic bilateral infarcts and persistent, but improved perivascular inflammatory changes of the celiac and renal arteries without recurrent or new dissection (Figure 2).

\section{Discussion and Conclusions}

Non-atherosclerotic non-inflammatory vasculopathies are uncommon with an overall combined incidence of $<0.03 \%$. $^{1,5}$ Segmental Arterial Mediolysis itself contributes $1 / 20$ th of this incidence $(0.001 \%)$. This incidence is distributed equally across all age groups, although there is a slight male preponderance. Hypertension is the most common preceding comorbidity reported, especially in cases of renal involvement. ${ }^{2,4,6-8}$ The pathogenesis leading to presentations of SAM is currently unknown, yet speculation ranges from embryological mesodermal defects ${ }^{9}$ to focal neuro-hormonal derangements resulting in vasospastic disease. ${ }^{1}$ Takagi et al speculated on embryological defects leading to the disease, based on the shared mesodermal origins of affected vessels and malignancies they observed in one patient. ${ }^{9}$

The vasospastic origin of SAM is speculatively based on theories of deranged trafficking of endothelins and catecholamines in smooth muscle wall-layers. Endothelins and catecholamines (epinephrine and norepinephrine) are potent vasopressors and endothelin-1 is 


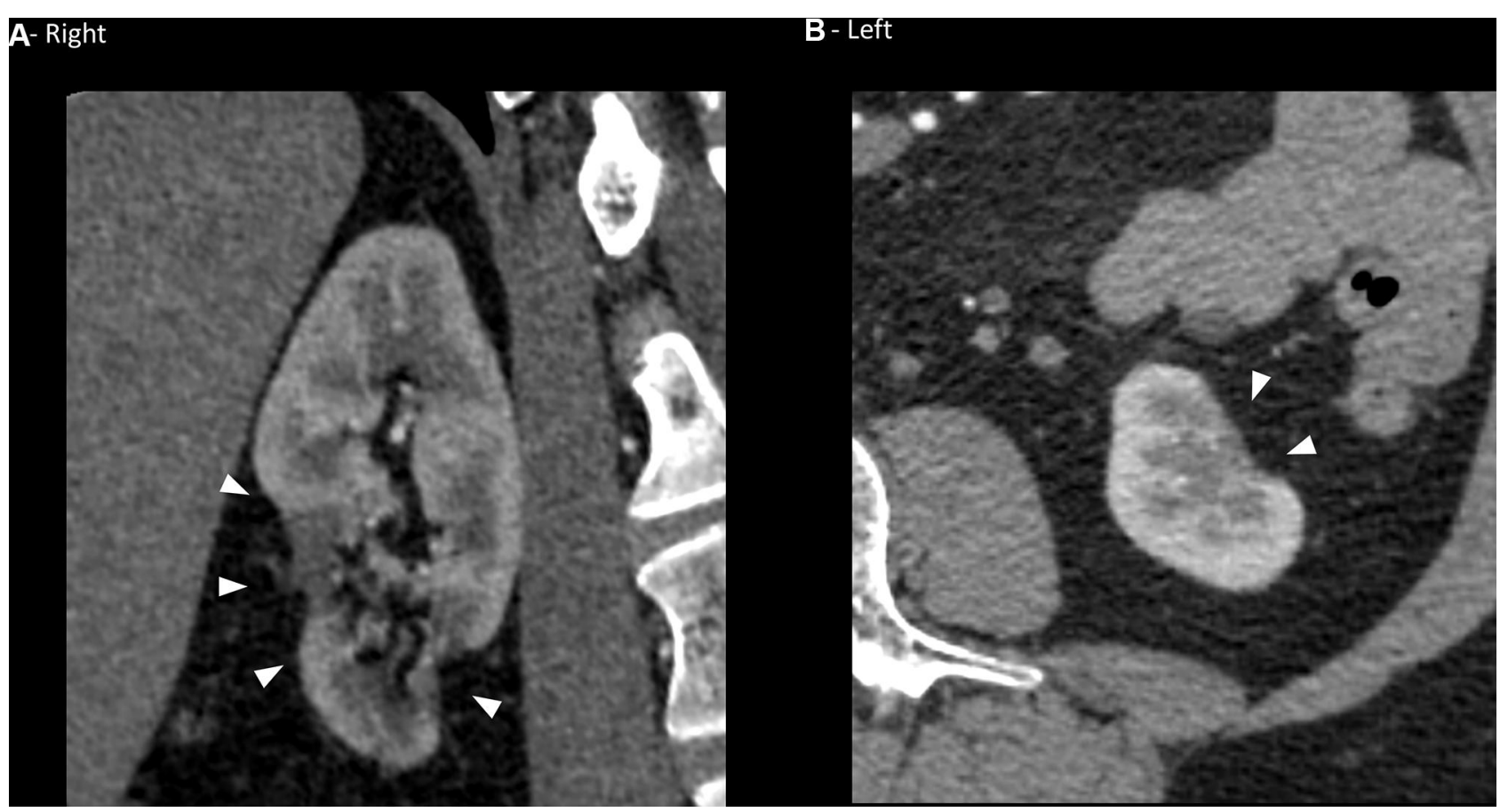

Figure 2 Renal CT angiogram 15 months after the initial insult. Demonstrates chronic infarcts and atrophy of the lower renal poles (arrowheads) of the right (A) and left (B) kidneys.

a known potentiator of these and other vasoconstricting mediators. ${ }^{10,11}$ Based on a histological study of tissue samples from 10 patients stained for Endothelin-1, Slavin et al have argued that endothelin dysregulation may be a cause of potentiation of other vasoconstrictors especially at the smooth muscle nerve terminals. ${ }^{12}$ This leads to a segmental constriction of the cells in the medial layer of arteries and results in the established histopathological sequence of the disease: micro-ischemia; vacuolization and micro-bleeds progressing to gaps and wall weakening; and consequently aneurysms, rupture and hemorrhage, dissection and thrombosis, or infarction. ${ }^{1,2}$ The vasospastic theory of SAM resonates with the erectile dysfunction (ED) seen in the subject of this case report as well as other co-morbid vasospastic diseases seen in the subjects of other case reports. ${ }^{12}$ Although ED is multi-factorial in origin, various studies implicate endothelin mediated vasoconstriction of the corpus cavernosum as a cause of the condition. $^{10,11}$

One or more vessels may be involved in the pathological process of SAM, with multiple vessels typically affected (46-85\%). ${ }^{1,13}$ The vessels most commonly affected in SAM include the celiac artery and branches $(80 \%)$, renal and cerebral vessels. ${ }^{2}$ The clinical presentation of SAM depends on which vascular beds have been dissected, thrombosed or infarcted, but chief complaints of abdominal, flank pain or headache predominate (with or without associated hematochezia, hematuria or stroke-like symptoms, respectively) and associated hypertension. ${ }^{2}$ Dramatic life-threatening hemorrhage into the abdomen, retroperitoneum, or brain can also be encountered.

Other causes of widespread vascular disease should be considered, including: systemic vasculitis; mycotic aneurysms; collagen vascular diseases; degenerative vasculopathies (eg, cystic medial necrosis and cystic adventitial artery disease); hereditary aneurysmal syndromes (eg, Lowey-Dietz syndrome); and non-atherosclerotic/noninflammatory vasculopathies (FMD and SAM). ${ }^{2}$ Workup for these differentials is clearly broad; however, Kalva et al describe a non-validated but convenient set of institutional diagnostic criteria used at Massachusetts General Hospital: Absence of atherosclerosis; absence of inflammatory markers (anti-nuclear antibodies, anti-neutrophil cytoplasmic antibodies, normal complement); absence of a clinical history suggesting another cause; absence of genetic markers for collagen vascular disorders; and segmental wall thickening, arterial dilatation, aneurysms, dissections, the "string-of-beads" appearance and absence of inflammation on imaging. ${ }^{6}$ In the reparative phase of the disease, fibrin and granulation tissue is deposited. This segmental thickening of arterial walls results in a stringof-beads sign (seen in about $30 \%$ of cases). ${ }^{14,15}$ 
After initial abdominal CT to rule out life-threatening causes of abdominal or flank pain, dedicated CT angiogram should be considered as the imaging modality of choice both for diagnosis and follow-up., ${ }^{2,16,17}$ Although histopathology is the gold-standard for diagnosis of SAM, it is usually unfeasible or not required given the efficacy of CTA. ${ }^{16}$

Controversy exists over the extent of disease in SAM. Slavin et al observed that generally only 2 or more arterial segments from the same or adjoining vascular beds may be affected. ${ }^{1}$ However, cases of multiple distributions are not uncommon. ${ }^{1,12}$ We agree with the supposition of other authors that subclinical SAM is likely to be manifested systemically by typical preceding comorbidities. We argue that this not only includes the commonly reported comorbidity of hypertension but also vasospastic diseases such as those previously reported as well as the erectile dysfunction seen in our case. ${ }^{12}$ Recognition of the potential for a wider extent of the disease than is symptomatically apparent arguably justifies wholebody radiographic exploration for lesions. ${ }^{3,18}$

Apart from a multi-disciplinary approach which should involve internal medicine, interventional radiology and vascular surgery, management of SAM depends on the presentation: stable vs unstable. In unstable presentations, patients should be resuscitated appropriately and may require emergent surgery for hemorrhage control. Some patients may also be candidates for endovascular therapy depending on the clinical situation but must be reasonably stable ${ }^{1,6,13}$ The conservative management of SAM in patients who are hemodynamically stable is controversial. Based on the high mortality rates of SAM generally and the high attribution of death to medical management in the case series they studied ( $82 \%$ ), some authors argue for the interventional approach (embolization) even for incidentally identified SAM lesions. ${ }^{8,13,19,20}$ In a non-systematic review of the literature on non-death outcomes of stable SAM cases at long-term follow-up (3 months to 102 months), we found that out of 37 cases conservatively (medically) managed, there was evidence of progression of this disease (death, recurrence, extension or new occurrence of aneurysms, dissection or infarction) in 14 patients $(\sim 38 \%)$. This was contrasted with progression in 9 out of 115 cases $(8 \%)$, that were invasively managed surgically or with endovascular interventions (stenting, coiling, angioplasty) (see Supplementary Materials). Among those cases managed with observation only, 17 out of 19 patients died (89\%). Although this is very poor quality evidence, it nevertheless lends support to invasive management for SAM in patients who are stable and are candidates.
The mainstay of medical management in stable patients is pain management, heart rate and blood pressure control to $<130 / 80 \mathrm{mmHg}$ and dual anti-platelet therapy (DAPT). ${ }^{6,21,22}$ Due to anti-inflammatory properties, statins may also impart benefit. Additionally, patients should be counselled to cease smoking. ${ }^{23}$ Although many care teams pursue anti-coagulation with heparin initially and during workup, this is usually ceased on diagnosis of SAM, given reports of recurrence as well as increased aneurysmal rupture with this therapy. ${ }^{3,6,18,23}$ Likewise, glucocorticoids should be avoided based on the case series of Lie and others who report the death of all SAM patients managed with glucocorticoids for presumptive vasculitis. ${ }^{3,24}$

The patient in this case report suffered a decline in renal function to CKD stage G2/3 within 9 months of initially presenting. This decline corresponded with CTA surveillance demonstrating stable bilateral renal infarcts and continued chronic vascular inflammation. The outcomes of long-term surveillance for SAM patients are under-reported with little information available regarding relevant sequelae beyond either the cessation of symptoms or radiographical evidence of disease stabilization. ${ }^{8,16}$ Although some patients have been followed for up to 9 years, most cases of reported SAM are followed-up for less than one year (see Supplementary Materials). As mentioned above, the majority of cases, including those with renal involvement, report resolution or at least no progression of the disease and it is widely reported to have a favorable prognosis. ${ }^{4,6,8,16}$ Nonetheless, clinical hypertension appears to be a not uncommon outcome and may indicate ongoing SAM related disease processes. This supports the argument made by other authors that SAM may represent a disease sitting on a continuum where FMD sits at the terminus. ${ }^{12,13}$ Similar to SAM, FMD is another non-atherosclerotic non-inflammatory vasculopathy. Whereas SAM presents with aneurysmal rupture, dissection and hemorrhage, FMD is known to present predominantly in middle-aged females with radiographical and clinical manifestations of arterial stenosis. Therefore, it is arguable that similar to hypertension, the CKD suffered by the patient in this report may be due to microvascular stenoses/occlusion that is not readily detectable on high-resolution CTA. Similar types of disease progression may be responsible for the consequent hypertension seen in other reports of SAM. To our knowledge, only 3 other cases of CKD associated with SAM have been reported. Unlike our patient, these three cases all involved patients with pre-existing co-morbidities. In two 
cases, CKD was present prior to the onset of the first event recognizably due to SAM. ${ }^{4,25}$ No information regarding the cause of CKD in those cases was reported. In the third case, the patient suffered from systemic sclerosis (SSc) and the decline in renal function was attributed to scleroderma renal crisis resulting from the combination of SSc and SAM. ${ }^{26}$

This is the first case report to our knowledge which describes CKD as a sequela of SAM without prior comorbidity. Although SAM is widely regarded as a disease with favorable prognosis, adverse sequelae such as chronic hypertension have been reported. It is the authors opinion that the instances of CKD and hypertension reported may be due to similar microvascular pathophysiology that is actually a characteristic of SAM progression. Further investigation on the long-term sequelae of SAM is required to gain a greater understanding of the relationship between SAM, FMD and associated co-morbidities.

\section{Abbreviations}

CKD, chronic kidney disease; SAM, segmental arterial mediolysis; SSc, systemic sclerosis.

\section{Data Sharing Statement}

The datasets and Supplementary Materials referred to in this case report are available from the corresponding author on reasonable request.

\section{Consent for Publication}

Written informed consent was obtained from the patient for publication of this Case report and any accompanying images.

\section{Acknowledgments}

The views expressed are solely those of the authors and do not reflect the official policy or position of the US Army, the Department of Defense, or the US Government.

\section{Author Contributions}

All authors made substantial contributions to conception and design, acquisition of data, or analysis and interpretation of data; took part in drafting the article or revising it critically for important intellectual content; agreed to submit to the current journal; gave final approval of the version to be published; and agree to be accountable for all aspects of the work.

\section{Disclosure}

The authors declare that they have no conflicts of interest.

\section{References}

1. Slavin RE. Segmental arterial mediolysis: course, sequelae, prognosis, and pathologic-radiologic correlation. Cardiovasc Pathol. 2009;18:352-360. doi:10.1016/j.carpath.2008.09.001

2. Pillai AK, Iqbal SI, Liu RW, Rachamreddy N, Kalva SP. Segmental arterial mediolysis. Cardiovasc Intervent Radiol. 2014;37:604-612. doi:10.1007/s00270-014-0859-4

3. Cohen DL, Soulen MC. A patient with acute kidney pain and high blood pressure. Clin J Am Soc Nephrol. 2015;10:696-702. doi:10.2215/CJN.10171014

4. Inada K, Maeda M, Ikeda T. Segmental arterial mediolysis: unrecognized cases culled from cases of ruptured aneurysm of abdominal visceral arteries reported in the Japanese literature. Pathol Res Pract. 2007;203:771-778. doi:10.1016/j.prp.2007.07.010

5. Ko M, Kamimura K, Ogawa K, et al. Diagnosis and management of fibromuscular dysplasia and segmental arterial mediolysis in gastroenterology field: a mini-review. World $J$ Gastroenterol. 2018;24:3637-3649. doi:10.3748/wjg.v24.i32.3637

6. Kalva SP, Somarouthu B, Jaff MR, Wicky S. Segmental arterial mediolysis: clinical and imaging features at presentation and during follow-up. J Vasc Intervent Radiol. 2011;22:1380-1387. doi:10.1016/ j.jvir.2011.07.001

7. Heritz DM, Butany J, Johnston KW, Sniderman KW. Intraabdominal hemorrhage as a result of segmental mediolytic arteritis of an omental artery: case report. J Vasc Surg. 1990;12:561-565. doi:10.1016/07415214(90)90011-X

8. Kim HS, Min SI, Han A, Choi C, Min SK, Ha J. Longitudinal evaluation of segmental arterial mediolysis in splanchnic arteries: case series and systematic review. PLoS One. 2016;11:e0161182. doi:10.1371/journal.pone. 0161182

9. Takagi C, Ashizawa N, Eishi K, et al. Segmental mediolytic arteriopathy involving celiac to splenic and left renal arteries. Intern Med. 2003;42:818-823. doi:10.2169/internalmedicine.42.818

10. Maas R, Schwedhelm E, Albsmeier J, Boger RH. The pathophysiology of erectile dysfunction related to endothelial dysfunction and mediators of vascular function. Vasc Med. 2002;7:213-225. doi:10.1191/1358863x02vm429ra

11. Ritchie R, Sullivan M. Endothelins \& erectile dysfunction. Pharmacol Res. 2011;63:496-501. doi:10.1016/j.phrs.2010.12.006

12. Slavin RE, Inada K. Segmental arterial mediolysis with accompanying venous angiopathy: a clinical pathologic review, report of 3 new cases, and comments on the role of endothelin-1 in its pathogenesis. Int J Surg Pathol. 2007;15:121-134. doi:10.1177/1066896906297 684

13. Tameo MN, Dougherty MJ, Calligaro KD. Spontaneous dissection with rupture of the superior mesenteric artery from segmental arterial mediolysis. J Vasc Surg. 2011;53:1107-1112. doi:10.1016/j. jvs.2010.11.034

14. Davran R, Cinar C, Parildar M, Oran I. Radiological findings and endovascular management of three cases with segmental arterial mediolysis. Cardiovasc Intervent Radiol. 2010;33:601-606. doi:10.1007/s00270-009-9651-2

15. Ro A, Kageyama N, Takatsu A, Fukunaga T. Segmental arterial mediolysis of varying phases affecting both the intra-abdominal and intracranial vertebral arteries: an autopsy case report. Cardiovasc Pathol. 2010;19:248-251. doi:10.1016/j.carpath.2009.02.002

16. Michael M, Widmer U, Wildermuth S, Barghorn A, Duewell S, Pfammatter T. Segmental arterial mediolysis: CTA findings at presentation and follow-up. AJR Am J Roentgenol. 2006;187:1463-1469. doi:10.2214/AJR.05.0281

17. Baker-LePain JC, Stone DH, Mattis AN, Nakamura MC, Fye KH. Clinical diagnosis of segmental arterial mediolysis: differentiation from vasculitis and other mimics. Arthritis Care Res. 2010;62:1655-1660. doi:10.1002/acr.20294 
18. Oki T, Adachi H, Tahara H, Kino S. [Spontaneous renal artery dissection with renal infarction: a case report]. Hinyokika Kiyo. 2011;57:611-614.

19. Chao CP. Segmental arterial mediolysis. Semin Intervent Radiol. 2009;26:224-232. doi:10.1055/s-0029-1225666

20. Soulen MC, Cohen DL, Itkin M, Townsend RR, Roberts DA. Segmental arterial mediolysis: angioplasty of bilateral renal artery stenoses with 2-year imaging follow-up. J Vasc Interv Radiol. 2004;15:763-767. doi:10.1097/01.RVI.0000133543.32123.DC

21. Britto MM, Lukies M, Milne C, Joseph T, Lee JC. Case of Segmental Arterial Mediolysis. BMJ Case Rep. 2018;2018. doi:10.1136/bcr2017-223731

22. Salifu MO, Gordon DH, Friedman EA, Delano BG. Bilateral renal infarction in a black man with medial fibromuscular dysplasia. $\mathrm{Am}$ J Kidney Dis. 2000;36:184-189. doi:10.1053/ajkd.2000.8292
23. Onteddu NK, Hindi Z, Rajashekar G, Kalva SP. Segmental arterial mediolysis presenting as spontaneous bilateral renal artery dissection. Radiol Case Rep. 2018;13:495-498. doi:10.1016/j.radcr.2017.11.017

24. Lie JT. Systemic, cerebral, and pulmonary segmental mediolytc arteriopathy: villainous masqueraders of vasculitis. Cardiovasc Pathol. 1996;5:305-314. doi:10.1016/S1054-8807(96)00071-3

25. Inada K, Ikeda T, Hayashi T. A Study on 20 cases of segmental arterial mediolysis (SAM) with multiple aneurysms, with a special reference to incidence, treatment and prognosis. Nihon Rinsho Geka Gakkai Zasshi. 2008;69:3101-3106. doi:10.3919/jjsa.69.3101

26. Kaneko S, Watanabe E, Abe M, et al. Scleroderma renal crisis with coexisting segmental arterial mediolysis presenting as intraperitoneal bleeding: a case report. J Med Case Rep. 2019;13:74. doi:10.1186/ s13256-019-1993-z

\section{Publish your work in this journal}

The International Journal of Nephrology and Renovascular Disease is an international, peer-reviewed open-access journal focusing on the pathophysiology of the kidney and vascular supply. Epidemiology, screening, diagnosis, and treatment interventions are covered as well as basic science, biochemical and immunological studies. The manuscript management system is completely online and includes a very quick and fair peer-review system, which is all easy to use. Visit http://www.dovepress.com/testimonials.php to read real quotes from published authors. 